\title{
Injustice et cruauté: la corrida constitutionnalisée
}

Commentaire de la décision "pro-corrida" du Conseil constitutionnel français du 21 septembre $2012^{1}$

\section{Sabine BRELS ${ }^{2}$}

Le 21 septembre 2012, l'estocade constitutionnelle a été portée à la cause "anti-corrida": la tauromachie est conforme à la Constitution. Alors que cette pratique est reconnue comme un acte de cruauté dans le code pénal, l'exception demeure là où "une tradition locale ininterrompue peut-être invoquée". Cette exception, contenue à l'alinéa 7 de l'article 521-1 du code pénal, est valable tant pour les combats de coqs dans les îles, que pour les corridas dans le sud de la France. C'est la constitutionalité de cette exception qui a été remise en cause devant le Conseil constitutionnel par deux associations: le "Comité radicalement anti-corrida" (CRAC Europe) et "Droits des animaux".

Sur le plan juridique, la décision rendue est contestable sur deux fondements principaux:

- Premièrement, elle cautionne au plus haut niveau juridictionnel une pratique légalement reconnue comme "cruelle" au nom de la seule "tradition";

- Deuxièmement, elle contredit ouvertement le principe constitutionnel d'"égalité" au nom d'une "exception" culturelle.

\section{$\underline{1-\text { La "cruauté" constitutionalisée au nom de la "tradition" }}$}

La décision du "Conseil des sages" est sans ambages: "La première phrase du septième alinéa de l'article 521-1 du code pénal est conforme à la Constitution ${ }^{\prime 3}$. En d'autres termes, le fait d'autoriser légalement des actes de cruauté de manière dérogatoire au principe de non-cruauté n'est pas anticonstitutionnel, tant qu'il existe une "tradition".

Le caractère "cruel" de la corrida n'est plus à établir, celui-ci étant reconnu implicitement dans le code pénal en tant qu'exception aux "sévices graves ou actes de cruauté envers les animaux". Quant à la notion de "tradition", les premières corridas n'ont été importées d'Espagne que depuis un siècle et demi et n'ont été légalisé qu'au milieu du XXe siècle ${ }^{4}$. Ainsi, la corrida a passé plus de temps à être une pratique illégale en France qu'une "tradition" légale dans le Sud du pays. Si jeune soit-elle, et bien que fermement protégée par ses défenseurs, cette "tradition" serait en déclin à en juger par la désaffection croissante des arènes d'année en année 5 . Quoi qu'il en soit, les "aficionados" n'ont jamais représenté la majorité de la population du Sud de la France.

\footnotetext{
${ }^{1}$ Décision $n^{\circ}$ 2012-271 QPC du 21 septembre 2012, en ligne: http://www.conseil-constitutionnel.fr/conseilconstitutionnel/francais/les-decisions/acces-par-date/decisions-depuis-1959/2012/2012-271-qpc/decision-n-2012-271-qpc-du-21septembre-2012.115564.html.

${ }^{2}$ Etudiante française au doctorat en droit, Université Laval (Québec), titulaire de la Bourse d'études supérieures du Canada Vanier. ${ }^{3}$ Id., Article 1er.

${ }^{4}$ Les premières corridas, apparues en 1853, étaient illégales en vertu de la "Loi Grammont" de 1850 qui interdisait de commettre publiquement des actes de cruauté envers les animaux. Ce n'est qu'à partir de la loi du 24 avril 1951 que l'exception pénale sur la corrida fut consacrée.

${ }^{5}$ Voir par exemple: Lucie Romano, "Corridas: Les aficionados boudent les arènes", 25 mai 2012, en ligne: http://www.20minutes.fr/societe/940567-corridas-aficionados-boudent-arenes.
} 
Ainsi, il semblerait que la notion de "tradition" soit moins jugée à l'aune de sa longévité et de la quantité de population qui la transmet ${ }^{6}$, qu'à son caractère "local" et "ininterrompu"7. Pourtant, l'exception pénale sur la corrida, qui s'applique à une minorité exempte de sanctions applicables partout ailleurs, devrait être acceptée par tous les citoyens français, malgré son caractère légalement et moralement contestable.

Puisqu' il est constitutionnellement consacré que la "tradition" peut constituer une exception légitime à la commission d'infractions, il y aurait alors matière à refondre le Code pénal! Par exemple, là où il existe une tradition mafieuse, le trafic et le crime organisés seraient alors légalement autorisés. Par extension, on entrevoit potentiellement les effets pervers d'une telle reconnaissance constitutionnelle...

Ailleurs dans le monde, d'autres régions et localités ont commencé à interdire la tauromachie, alors même qu'il s'agissait d'une tradition ancrée: l'Espagne, berceau de la corrida, a même été le premier à montrer l'exemple à travers l'interdiction de la corrida en Catalogne depuis 2010 (et à Saint-Sébastien pour 2013), suivie en Amérique latine par Bogota en Colombie et Lima au Pérou depuis l'été 2012.

Toutefois le Conseil constitutionnel ne traite ni de "cruauté" ni de "tradition" dans sa décision, mais simplement de la question de constitutionalité de l'exception de l'alinéa 7 de l'article 521-1 en regard du principe d"'égalité" des citoyens devant la loi, tel qu'allégué par les requérants.

\section{$\underline{\text { 2- L'"inégalité" constitutionalisée au nom de l'"exception" }}$}

Le principe d'égalité devant la loi est énoncé à l'article 6 de la Déclaration des droits de l'homme et du citoyen de 1789 en ces termes: "La loi [...] doit être la même pour tous, soit qu'elle protège, soit qu'elle punisse".

Selon les associations requérantes, l'exception prévue par le code pénal pour les corridas porterait atteinte à ce principe ${ }^{8}$. Au contraire, le Conseil constitutionnel décide que l'exception tauromachique lui est conforme ${ }^{9}$. Ainsi, le fait d'autoriser la commission de délits à certains individus tout en punissant tous les autres, ne serait pas contraire au "principe d'égalité" des citoyens devant la loi. Dans les considérants énoncés par le Conseil dans sa décision, les justifications rationnelles à cette contradiction semblent toutefois faire défaut. En effet, il semblerait que cette inégalité citoyenne soit constitutionalisée plus en vertu de considérations de "fait" que de "droit". En ce sens, ces considérations tiennent plus à une conjecture politique favorable à la tauromachie qu'à une argumentation juridique rationnelle. Plus que jamais, il est ainsi possible d'affirmer que: "Les juridictions qui se sont prononcées jusqu'à ce jour ont manifestement fait ouvre un peu subjective et partisane de la corrida" ${ }^{10}$.

\footnotetext{
${ }^{6}$ Selon le dictionnaire commun, deux éléments semblent déterminants à la définition de la "tradition": l'usage perpétré "sur un long espace de temps" et la "transmission" intergénérationnelle commune. Voir le Larousse en ligne:

http://www.larousse.com/en/dictionnaires/francais/tradition/78903.

${ }^{7}$ En effet, il semblerait que les décisions de justice rendues sur cette exception ce soit plutôt intéressées à l'interprétation de ces adjectifs qu'à la légitimité même du concept de "tradition" pour légaliser par exception la commission d'infractions. Voir le Commentaire de la Décision $n^{\circ}$ 2012-271 QPC du 21 septembre 2012, Association Comité radicalement anti-corrida Europe et autre, (Immunité pénale en matière de courses de taureaux), en ligne: http://www.conseil-constitutionnel.fr/conseilconstitutionnel/root/bank/download/2012271QPCccc_271qpc.pdf.

${ }^{8}$ Considérant 2 de la Décision $n^{\circ}$ 2012-271 QPC du 21 septembre 2012, en ligne: http://www.conseil-constitutionnel.fr/conseilconstitutionnel/francais/les-decisions/acces-par-date/decisions-depuis-1959/2012/2012-271-qpc/decision-n-2012-271-qpc-du-21septembre-2012.115564.html.

${ }^{9}$ Id., Considérant 4.

${ }^{10}$ Voir sur le site du CRAC Europe, "L’article 521-1 vu par Gérard CHAROLLOIS" (Vice-président au tribunal de grande instance de Périgueux et Président de la Convention Vie et Nature pour une Ecologie Radicale), en ligne: http://www.anticorrida.com/-Une-activite-legale-.html?page=article_imprime\&id_article=98.
} 
La décision rendue comprend une autre dimension inquiétante: l'exception culturelle peut primer sur la règle morale en matière pénale. Alors que la règle morale, de "bon sens", prohibe les actes de cruauté envers les animaux ${ }^{11}$, l'exception culturelle l'autorise. Mais alors que la norme pénale est en principe valable pour tous, il semblerait qu'une exception traditionnelle puisse légitimer l'action délictuelle. Seraitce désormais l'exception qui confirme la règle pénale? Si oui, comme semble l'entériner cette décision constitutionnelle, il y a sans doute de quoi crier à l'insécurité juridique!

Au lieu de s'inscrire dans une évolution morale favorable à la protection animale, la France semble se complaire dans une rétrogradation illimitée à ce sujet. En effet, alors que le "Conseil des sages" aurait eu une belle occasion de mettre un terme à cette tradition cruelle, il n'a pas décidé de faire primer la moralité sur la cruauté. Une décision scandaleuse du point de vue éthico-légal, mais toutefois prévisible dans un pays à la gouvernance partisane. Pourtant, les animaux sont légalement reconnus comme des êtres sensibles $^{12}$ et la corrida comme un acte cruel. Mais la souffrance gratuite et l'inégalité citoyenne sont désormais cautionnées par le plus haut degré de la légalité, le niveau constitutionnel, pour la plus grande satisfaction de quelques uns. Ainsi en est-il décidé: l'injustice est constitutionalisée! Tant pour les taureaux que pour les citoyens français...

\footnotetext{
${ }^{11}$ Plus précisément, l'article 521-1 du Code pénal interdit les "sévices graves ou actes de cruauté" envers tout "animal domestique, ou apprivoisé, ou tenu en captivité" sachant que les animaux sauvages sont exclus de cette protection.

${ }^{12}$ Article L.214-1 du Code rural: "Tout animal étant un être sensible doit être placé par son propriétaire dans des conditions compatibles avec les impératifs biologiques de son espèce".
} 